\title{
Research on Parametric Modeling Optimization of Trajectory Correction Projectile Based on FVM
}

\author{
Wei ZHOU, Jian ZHANG*, Qingjie NI, Yufeng HAO, Yu LIANG
}

\begin{abstract}
The solution of fluid computer based on N-S equations is the core of Computational Fluid Dynamics (CFD), and Finite Volume Method (FVM) is the computational method of the main stream of fluid. The grid technology closely related to CFD is only a foundation, and it becomes more and more lagging with the development of CFD in computer. But grid is the most important and indispensable part. For the fluid aerodynamic simulation computation, the grid section is popular now hexahedral meshing method, the meshing time of some complex models tends to account for about $90 \%$ of total amount control. Although it is the basic work, the proportion of the workload is large amounts. Therefore, the parametric modeling can substantially reduce some unnecessary modeling time. For parametric modeling, VB, VC and other programming languages are generally used to parametric development model. Now a great number of commercial software that has integrated this function has no use for additional development. In this paper, the parametric functions are applied to the simulation model in the design, focusing on the analysis of parameterized system design script problems, which were liable to occur for the correction projectile and other related ammunition aerodynamic design provides a good research ideas.
\end{abstract}

Keywords: parametric; aerodynamics; correction projectile

\section{INTRODUCTION}

Intelligent ammunition, with its precise strike capability, plays an increasingly important role in the modern local wars [1]. However, in the development of intelligent ammunition, a number of pre-simulation verifications is necessary, including the establishment of an external ballistic model, the analysis of aerodynamics impact on the steering gear structure, the servo correction capability in pneumatic environments, etc.

The current CFD and the software which applies its theory, play an important role in the field of ballistic aerodynamic research of projectiles [2, 3]. Excellent aerodynamic model can reduce the calculation error to improve precision of the software, following with the increase of the model meshing workload, usually tend to occupy the entire aerodynamic simulation calculation of about $80 \%$ of the workload, some complex grids are accounted for more than $90 \%$ [4]. Because the core of CFD is the computer solution of fluid as the development of computer, the fluent methods of solution are closer to reality.

The grid has always been considered as the basic role in the process of computing. But the quality of mesh affects the accuracy of the calculation results directly. The full use of computational fluid dynamics on the computer can only be accomplished by the complex shape of the grid. In other words, it is more difficult to generate reliable flow field grids with complex shapes than flow field technology. Now a number of commercial software has integrated this function without additional development along with the development of computers and related software. Therefore grid technology is one of the key technologies of CFD.

In addition, in the process of aerodynamic simulation, it is necessary to optimize the aerodynamic design of scheme of multiple, not only to design and calculate the aerodynamic layout for the entire projectile, but also to optimize the key aerodynamic structure of the projectile in a localized manner [5]. Especially the simulation computation of the local modification scheme, a lot of design time was used to carry out repeated modeling and meshing [6]. It is of great significance to apply the parametric design idea to the aerodynamic development of smart ammunition. Parametric design provides a new concept for ammunition design that can save the modeling process of intelligent ammunition, reduce the complexity of repeated work and greatly drop off the design cycle. For parametric simulation design $[7,8], \mathrm{VB}, \mathrm{VC}$ and other programming languages are generally used to parametric development model. However, as the commercialization of fluid computing software becomes more sophisticated, parametric modeling functions in software development have been integrated by software vendors and are greatly improving the performance of the use of software.

So far many commercial software platforms modularize and parameterize different simulation technologies. It can be used to complete data lossless transmission through analytical tools by data correlation. For example, the parametric design of rotary locking mechanism of automatic rifle was carried out based on the secondary development of UG [9]. Its own script generation tool can perform script generation for complex models. Combining parametric design ideas and parametric + script generation functions, it can greatly improve the design efficiency of intelligent ammunition and provide a good design basis for the aerodynamic analysis of CFD. This article focuses on parametric design analysis of mesh module.

\section{PARAMETRIC DESIGN PRINCIPLES}

For the influence of canard angle on the aerodynamic characteristics of 2D trajectory correction projectile, the parametric design is a better choice. The flow chart of the parametric design idea is shown in Fig. 1, which is different from the traditional programming language. The local model of the complex model is first established by using the three-dimensional modeling software, and then the model was modified when imported into design module. By using the geometric transformation of design model and Boolean calculation methods to parametric design of the local construction for research that is in need, and then the script design and the parametric setup of grid are completed. Finally, the data is imported into fluid 
simulation software to complete the simulation calculation. When analysing the results of numerical calculation, the working efficiency increased by more than dozens of times.

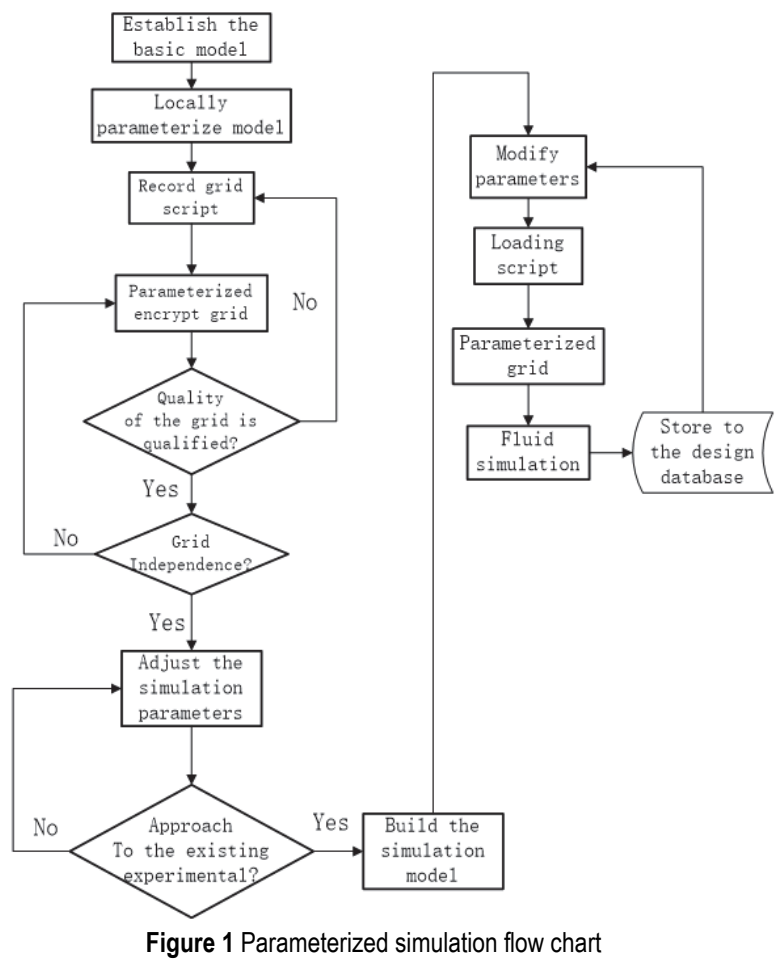

\subsection{Model Parameterization}

In this paper, the aerodynamic design of trajectory correction projectile is designed based on a certain type of projectile, its two-dimensional drawing is shown in Fig. 2.

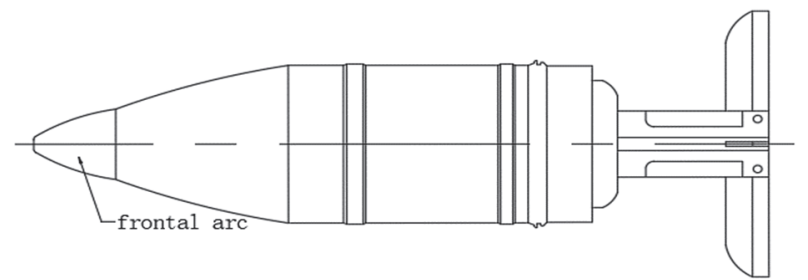

Figure 2 The two-dimensional drawing of a certain type of projectile

Table 1 Parameterized design data of the frontal arc of the projectile / $\mathrm{mm}$

\begin{tabular}{|c|c|c|c|c|c|}
\hline Scheme & $\begin{array}{c}\text { O.d of } \\
\text { head }\end{array}$ & $\begin{array}{c}\text { Front } \\
\text { arc }\end{array}$ & $\begin{array}{c}\text { Cylindrical } \\
\text { part }\end{array}$ & $\begin{array}{c}\text { Length of } \\
\text { canard }\end{array}$ & $\begin{array}{c}\text { Height of } \\
\text { canard }\end{array}$ \\
\hline 1 & 10 & 200 & 50 & 20 & 14 \\
\hline 2 & 11 & 260 & 60 & 24 & 15 \\
\hline 3 & 12 & 320 & 70 & 28 & 16 \\
\hline 4 & 13 & 380 & 80 & 32 & 17 \\
\hline 5 & 14 & 440 & 90 & 36 & 18 \\
\hline
\end{tabular}

Using the advantage of design module of software is compatible with most of the current commercial 3D software. The main part of the type of projectile is simplified and designed by 3D software, such as UG, SolidWorks, PRO/E and so on, only the main aerodynamic characteristic of projectile is preserved, and is parameterized design to the frontal arc of the projectile. As shown in Fig. 2. parametric analysis of 5 schemes was according to the simulation results, using linear interpolation method to optimize the following scheme. The initial stage of the size structure variables is shown in
Tab. 1, select one for modeling diagram shown in Fig. 3.

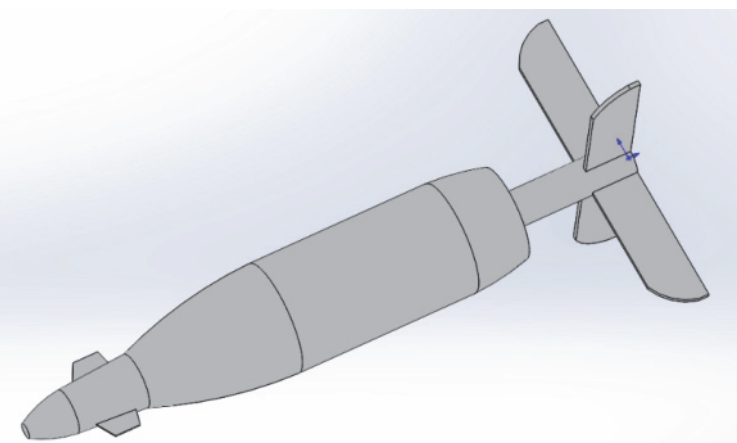

Figure 3 The model in 3D software

Then the model is imported into design module for the post processing, modeling the local model of the correction agency that is needed to research in DM, and all possible parameter schemes are parameterized, for example, the shape of the fuse head, the shape of the canard rudder, the position of the canard rudder and the deviation of the canard rudder angle, etc. Fig. 4 shows the position and shape change of canard rudder under the position change of canard rudder, the deflection angle of canard at 6 degrees and 6 degrees negative. Finally, the canard is combined into a whole model by Boolean operation and output to block and grid processing.

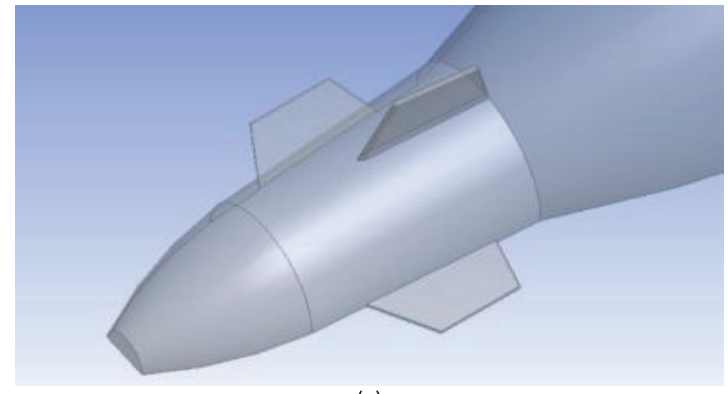

(a)

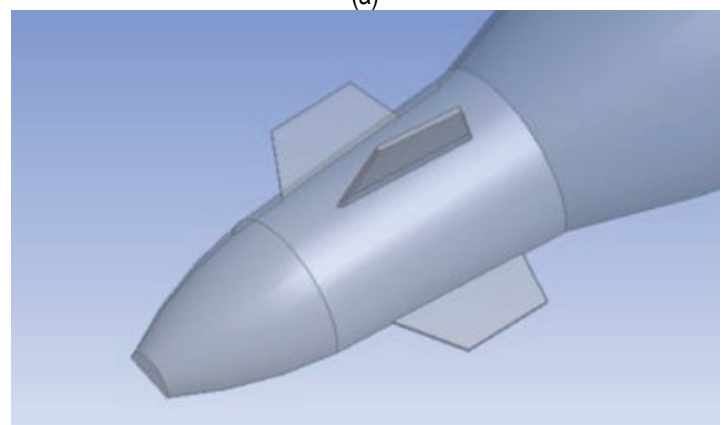

(b)

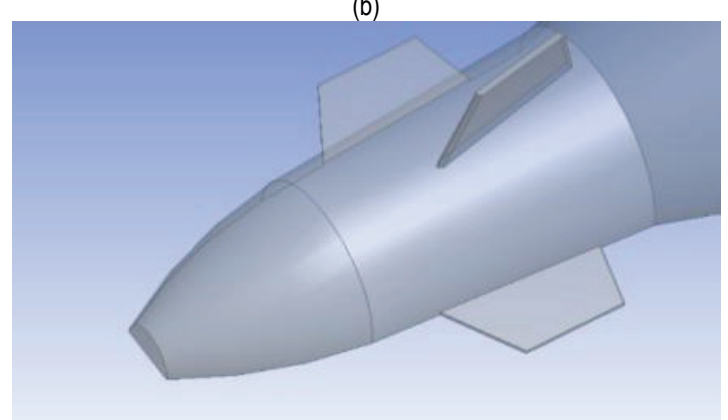

(c)

Figure 4 The change of the position and shape of the canard under different parameters: (a) axial position offset of duck rudder; (b) 6 degree deflection angle and (c) 6 degree deflection angle 


\subsection{Model's Script Edit and Grid Parameterization}

Because the projectile's motion of exterior trajectory single direction in the case of instantaneous and hexahedral meshes has very high calculation accuracy of simulation for single direction flow problem, therefore using it to mesh the model.

The space outside the model is divided into high quality hexahedral grid by using block function. A script running program can store the operation process of a block in the command flow script. We can quickly generate blocks by invoking scripts when making complex model changes, which greatly reduces the blocking time after modeling. The scripting commands is written by $T_{\mathrm{cl}} / T_{\mathrm{k}}$ (Tool Command Language/Tool Kit) tool control language, which is a programming language of string, and all script commands start with IC, such as command:

ic hex split grid $28340.4 \mathrm{~m} \mathrm{~A} 1 \mathrm{~A} 2$

It indicates that the grid is divided at the location of 0.4 (normalization of the distance of the point 28, 34) which the distance form 28 , and the line passes through the A1 and the A 2 parts. The creation and modification of complex model scripts can be completed by modifying, deleting and increasing the scripting language. Finally, the grid of block is densified through the grid parameterization, and using the function relation of point distance between vertexes.

\section{SCRIPT DESIGN AND PROBLEM ANALYSIS}

\subsection{Problems which are Easily Caused in Scripting Design}

The node number of the model remains unchanged when the characteristics of 2D trajectory correction projectile are changed slightly. At this time, the script of meshing can complete the meshing instantly. However, there will be many problems when dividing the script, which requires a lot of time to check the script to solve these problems, so it is necessary to analyze some problems of the script in detail.

For example, grid distortion often occurs in the process of design and running script, as shown in Fig. 5.

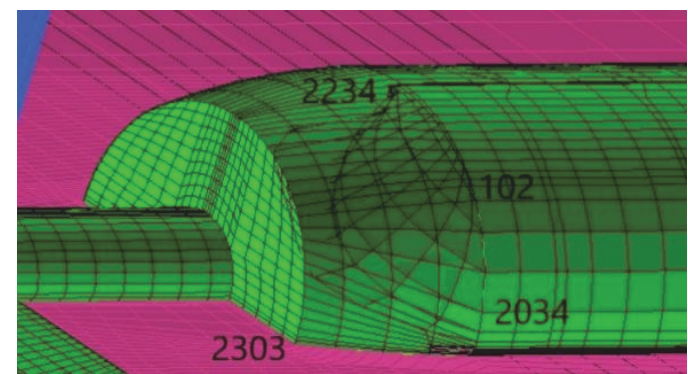

(a)

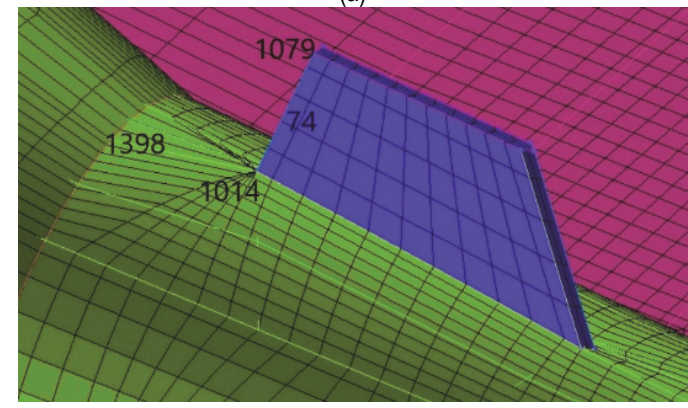

(b)

Figure 5 Mesh distortion produced in the process of generating grid: (a) projectile tail mesh distortion and (b) same direction mesh distortion
There is an error in the script running, program termination, as follows:

(Error in replay of ic_split_grid 346129 MODEL 0:0.2 m DANTI WALL IN OUT

Usage split_grid node dim val[dim:min,max $][\mathrm{mpar}][\mathrm{m}$ $\mathrm{m} 1 \mathrm{~m} 2 \ldots]$

Error in replay script)

The script file on the GUI update, BoCo settings, undo $\log$ and other script statements, these statements are of no practical significance. Such as the cancel order of undo:

ic_undo

ic_uns_update_family_type

visible $\{$ CREATED_FACES YADUO WEIYI\}

These are three kinds of problems that often appear in script design. Once there are problems, we need to check the script's command line, eliminate problems one by one, and increase the workload of dividing block.

\subsection{Analysis of the Cause of Script Design}

The reasons for the above problems are summarized as follows:

1) Inadequate understanding of grid computing

All the CFD data transmission is made up of nodes in the grid. The grid does not disappear in the visual operation environment. And the reason is that the mainstream fluid calculation software nowadays is based on N-S equations, and the finite volume method is used as the basic data representation form. Each region is divided into integral forms to express the volume in the region, and further the fluid calculation is carried out. Fig. 6 shows that the lightcolored line in the middle area on the right represents the hidden grid, which actually exists. That comes out of the actual mesh which is often hidden in the process of operation in order to simplify and visualize mesh generation, and the actual mesh has not disappeared, but is no longer visualized. However, these computed grids are indispensable nodes in numerical simulation, and they transfer information on these grids. The dotted line region is the integral region of the FVM.

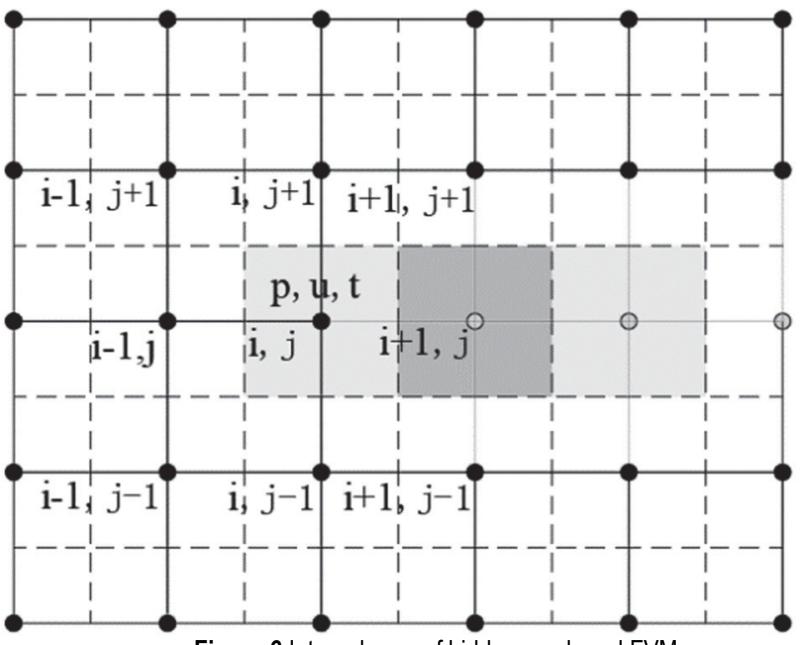

Figure 6 Integral area of hidden mesh and FVM

The adjacent nodes can be automatically generated according to the very orderly number of grid nodes; the mesh is generated in short time with the simple structure and good consistency; it is easy to load the boundary. 
Its disadvantage is that it only applies to the shape rule model in the past, and the orthogonal structured grid can be applied to the calculation of irregular air flow along with the development of technology. The model grid is shown in Fig. 7.

The whole calculation of mathematical expression is linear and continuous, and the solutions of differential and integral equations are also continuous. But, the numerical solutions of calculus equation only have definite values at specific discrete points in some regions. These values of temperature, pressure and velocity are calculated by the relationship between nodes. They are given below in Eqs. (1) and (2).

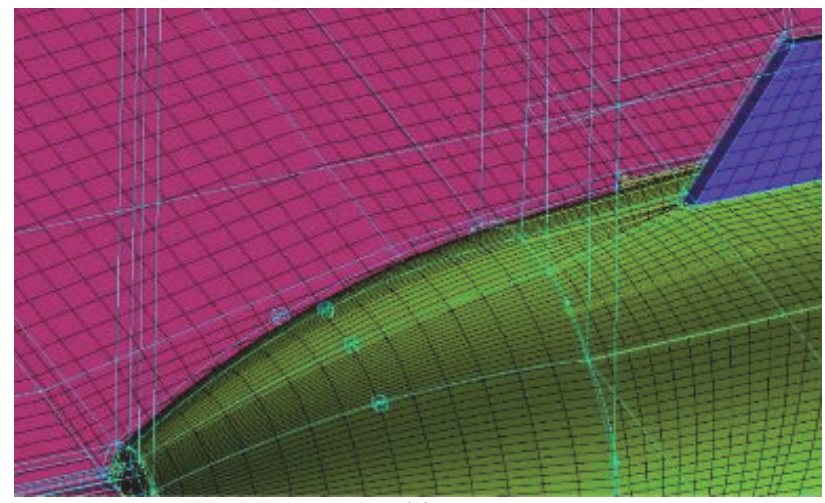

(a)

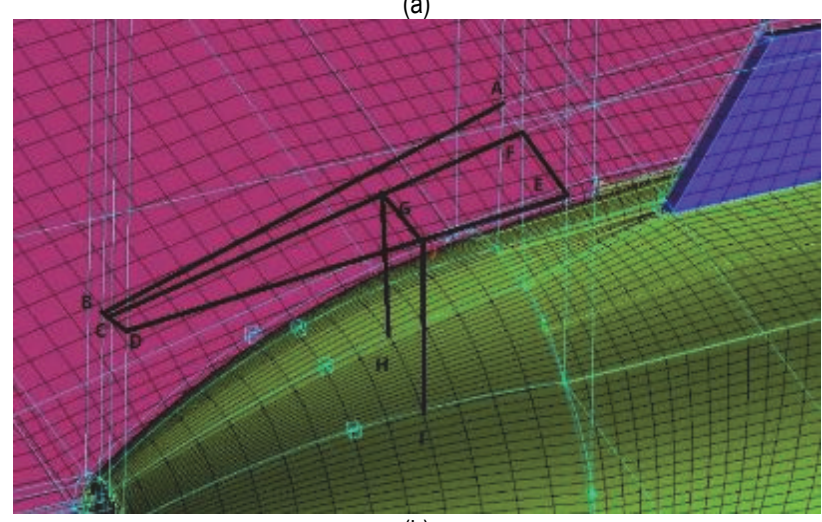

(b)

Figure 7 Hidden meshes in three-dimensional meshes: (a) hidden grid and (b) partially displays hidden grid

$\frac{\partial}{\partial t}\left(\int_{\Delta V} \rho \phi \mathrm{d} V\right)+\int_{A} \vec{n}(\rho \phi \vec{V}) \mathrm{d} A=\int_{A} \vec{n}(\Gamma \phi) \mathrm{d} A+\int_{\Delta V} S_{\phi} \mathrm{d} V$

Steady state diffusion problems:

$$
\int_{A} \vec{n}(\Gamma \nabla \phi) \mathrm{d} A+\int_{\Delta V} S_{\phi} \mathrm{d} V=0
$$

where $\phi$ is the rate of increase of inside the control volume. $\rho$ is the density of the air in the incoming flow, $V$ is the volume of the incoming flow, $S$ is the source term, $\Gamma$ is the diffusion coefficient, $A$ is the area of the volume interface. $n$ is the component of vector in the direction of normal to surface element $\mathrm{d} A$.

The $\mathrm{C}$ or O-shaped grid can be formed along the physical boundary according to the surface of the flying object, and the quadrilateral or hexahedron structure of the whole grid can be maintained. And the two-dimensional quadrilateral mesh is generated in a plane perpendicular to the axis of the projectile. Two-dimensional quadrilateral meshes with different cross-sections also form quadrilateral meshes in the circumferential direction, finally forming three-dimensional hexahedral meshes, as shown in Fig. 8.

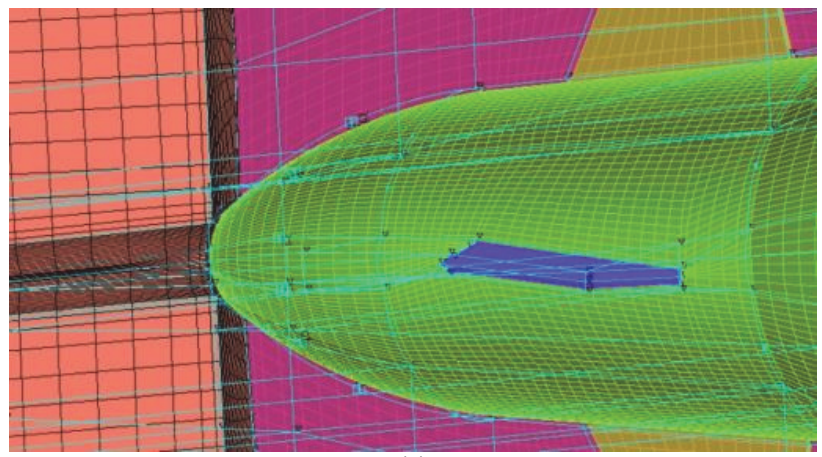

(a)

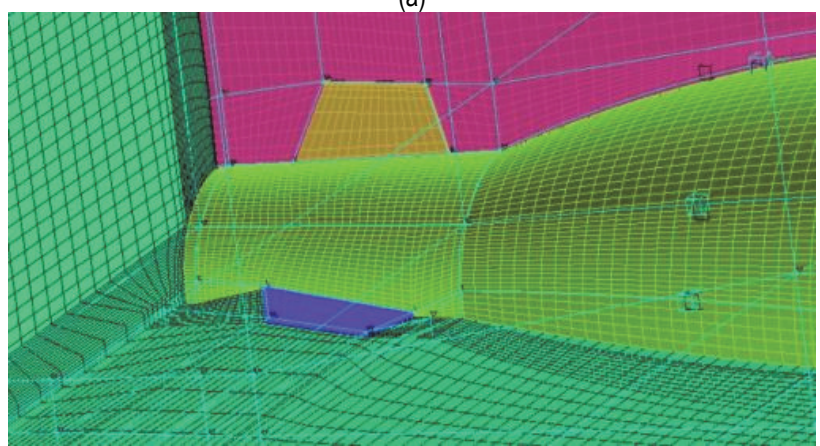

(b)

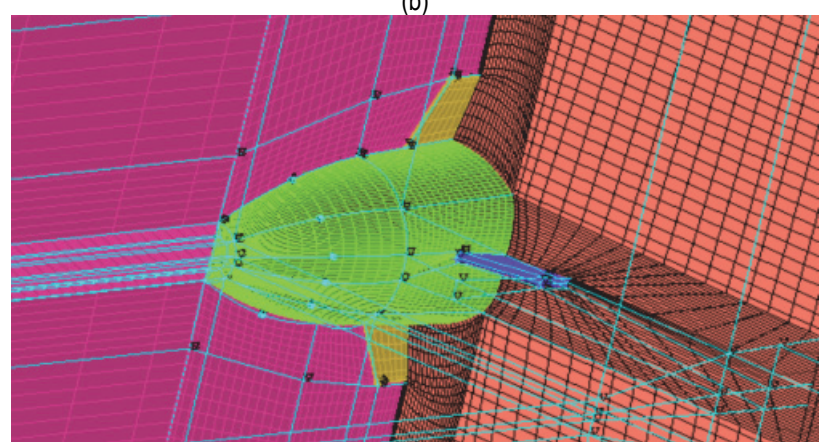

(c)

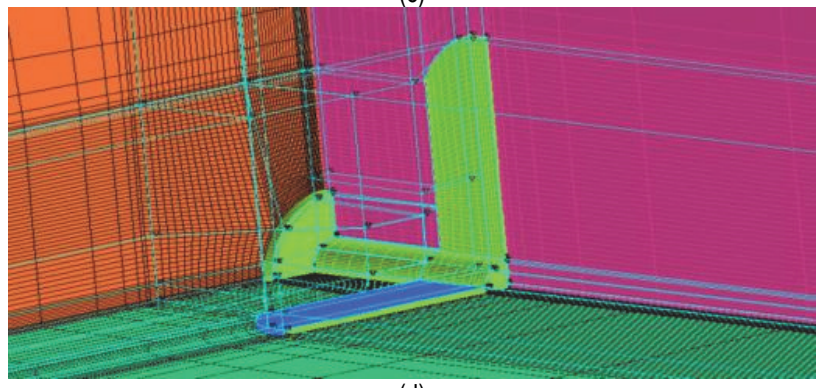

(d)

Figure 8 Hexahedral meshes around projectiles: (a) fuze head and (b) fuze front arc and (c) duck rudder and (d) projectile tail

Fig. 9 shows the cross-sectional and three-dimensional distribution of hexahedral meshes. It can be seen that the hexahedral meshes are uniformly distributed, and the mesh is refined on the surface of the object where the surface changes greatly. The spatial grid has good orthogonality, so that the mesh points can be expanded along any direction without needing to go straight up or down in the orthogonal direction. 

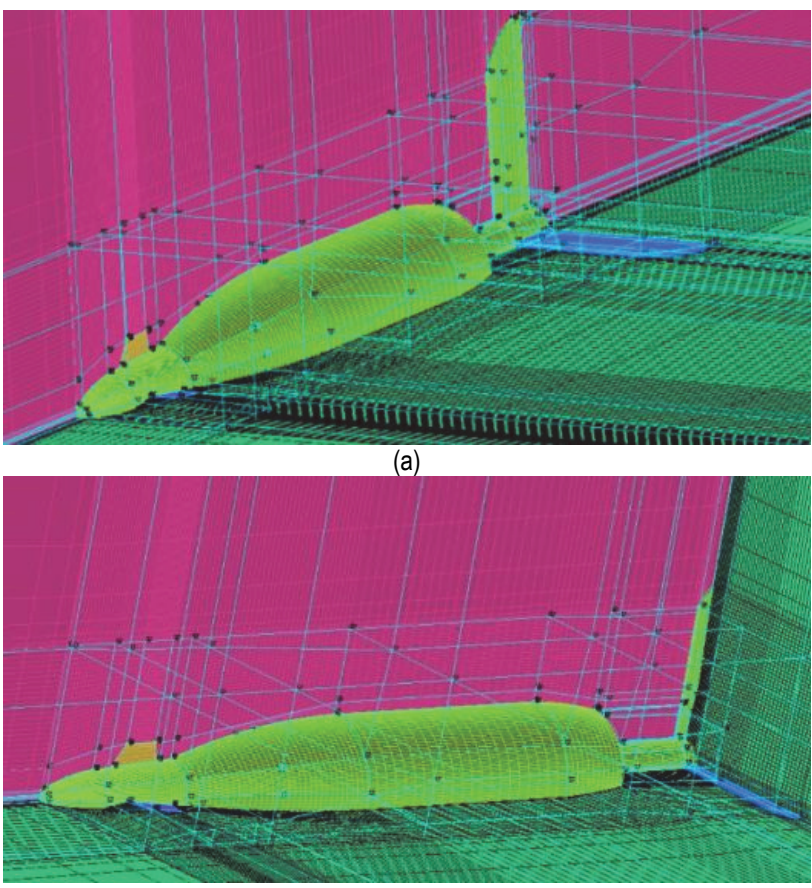

(b)

Figure 9 mesh distribution of projectile: (a) radial plane and (b) axial plane

2) Nonstandard block operation

The Fig. 5 problem is taken as an example, because the mapping point in the command has a problem in the process of operation.

ic hex_set_edge projection 20342303 (\#2303 should be 2234) 01 EDGE102

ic hex set edge projection 13981014 (\#1014 should be 1790) $0 \overline{1}$ EDGE74

The lines between points 2034 and 2303 have mapping relations with line 102, and the lines between point 1398 and 1014 have mapping relations with line 74 . This error is due to the script command generation, and it is impossible to make a subsequent modification of the grid by removing and reestablishing the mapping relationship.

The reason for this mistake is that the correct line segment was not selected in the visualization operation. The system chooses the wrong line segment according to the proximity principle, and the script only can record the information of the point. Therefore, we must standardize operation procedure when doing script generation operation, and the script commands are monitored at any time, and the analysis and record are carried out carefully.

3) Redundant invalid commands in the script

The core function of script statements is the operations on the block and the grid, each command is the default operation command of the system, system cannot distinguish for invalid command. As follows:

Error in replay of ic_split_grid 346129 MODEL 0:0.2 m DANTI WALL IN OUT

It records script error which caused the termination of programs, mainly as a result of block cutting, and repeated selection and revocation operations in the process of merging and mapping, and invalid commands were not deleted, and select the point and the line is not standardized. Therefore, we should pay attention to the timely removal of redundant operations in the script controller during the synchronization of block operations and generation scripts. For example, the following command:

ic_uns_subset_add_families_and_types uns_sub_0
BLOCK all

This is an invalid command, because we select line cross point which should not be crossed during the operation, and the system hints cannot be selected, therefore, it is a command statement that failed to operate. But the script also recorded the wrong operation, it will terminate the script situation when running the script. Such commands need to be removed in time when the scripts are recorded.

In addition, because of the convenience of visual operation and the specific cohesive function of the block, some areas are artificially hidden when they are divided into blocks for a particular operation. But the point has not disappeared and turned into a hidden point in the division of the whole region. Because of the existence of hidden points. The script command completes the command generation with the value of the hidden point, which is a frequent occurrence of script editing.

The local mesh of the new geometric model is separated from the original one when the local part of the model is redesigned. Model parameters and grid parameterization can be linked up through script design. Thus, a series of data can be obtained efficiently, which saves the complex operation of programming and is easy to understand.

\subsection{Mesh Parameterized Dividing}

The geometric characteristics of the projectile nose for key research. Using the corresponding point distribution function rule, the block is encrypted by parameterized grid. For example, a biexponential and exponential function are used to perform a point distribution operation. It can solve the problem of distribution and aggregation of the vertex and the arc degree of the projectile nose. The expression is below in Eqs. (3) and (4).

Biexponential

$S_{i}=\int_{0}^{i} \exp \left(a_{0}+a_{1} x+a_{2} x^{2}+a_{3} x^{3}+a_{4} x^{4}\right) \mathrm{d} x$

Exponential

$S_{i}=P \mathrm{e}^{R(i-1)}$

where $S$ is the distance from the starting point to the node $i, i$ is a point on the line, $P$ is the distance between the first two points of the beginning or the terminal, $R$ is ratio. The distribution function of the biexponential point is to determine the order in the formula according to the ratio of $R$, so as to adjust the distance between points. The order is zero and it is a linear equation when $R$ is zero.

\subsection{Grid Quality Checking}

Specific mesh encryption methods are selected to mesh blocks and parameterize them, and check the quality of determinant $2 \times 2 \times 2$, determinant $3 \times 3 \times 3$ and angle, as shown in Fig. 10.

The figure below shows that the shape of each hexahedral mesh and the location of each node in space affect the quality of the mesh. The corresponding matrix 
can be established according to the position and order of the shape function. The specific values and the relationship between Jacobian matrix and standard hexahedron is obtained through transforming six surfaces and standard hexahedron. That is to say, the real hexahedron deviates from the ideal hexahedron. The Jacobian ratio of $2 \times 2 \times 2$ $=8$ nodes ranges from 0 to 1 , and that of $3 \times 3 \times 3=27$ nodes ranges from -1 to 1 .

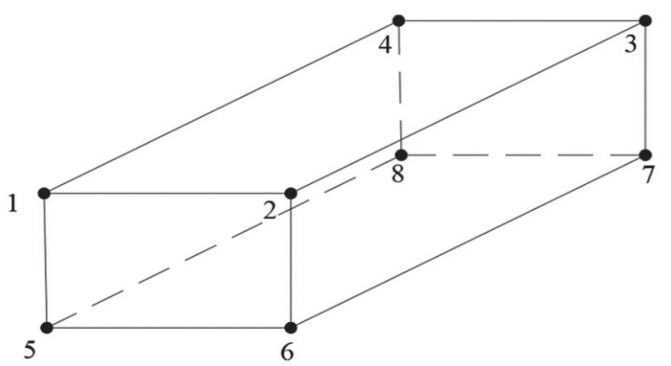

(a)

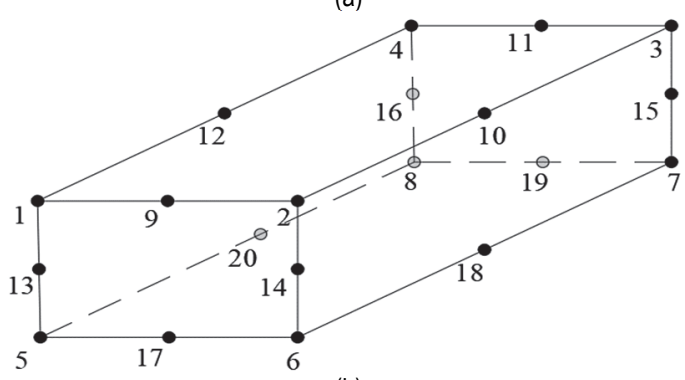

(b)

Figure 10 checking the quality of determinant: (a) determinant $2 \times 2 \times 2$ and (b) determinant $3 \times 3 \times 3$

The shape of the ideal hexahedron unit is shown in Fig. 10 a when the value is 1 , the $X$-axis is horizontal, the $Y$-axis is vertical to the plane, and the $Z$-axis is vertical to the plane. The same is true of determinant $3 \times 3 \times 3$. The essence of grid quality inspection is to transform the matrix value of irregular hexahedral elements into the actual shape in standard coordinates by coordinate transformation and mapping. The Jacobian matrix of $2 \times 2 \times 2$ is given below in Eq. (5)

$$
[\boldsymbol{J}]=\left[\begin{array}{llll}
\frac{\partial N_{1}}{\partial x_{1}^{\prime}} & \frac{\partial N_{2}}{\partial x_{2}^{\prime}} & \cdots & \frac{\partial N_{s}}{\partial x_{s}^{\prime}} \\
\frac{\partial N_{1}}{\partial y_{1}^{\prime}} & \frac{\partial N_{2}}{\partial y_{2}^{\prime}} & \cdots & \frac{\partial N_{s}}{\partial y_{s}^{\prime}} \\
\frac{\partial N_{1}}{\partial z_{1}^{\prime}} & \frac{\partial N_{2}}{\partial z_{2}^{\prime}} & \cdots & \frac{\partial N_{s}}{\partial z_{s}^{\prime}}
\end{array}\right]\left[\begin{array}{llll}
\frac{\partial N_{1}}{\partial x_{1}} & \frac{\partial N_{2}}{\partial x_{2}} & \cdots & \frac{\partial N_{s}}{\partial x_{s}} \\
\frac{\partial N_{1}}{\partial y_{1}} & \frac{\partial N_{2}}{\partial y_{2}} & \cdots & \frac{\partial N_{s}}{\partial y_{s}} \\
\frac{\partial N_{1}}{\partial z_{1}} & \frac{\partial N_{2}}{\partial z_{2}} & \cdots & \frac{\partial N_{s}}{\partial z_{s}}
\end{array}\right]^{-1}
$$

Choosing the mesh encryption method to mesh and parameterize the blocks. All grid values greater than 0.6 mesh angle greater than 13.5 degrees through Determinant $2 \times 2 \times 2$ and Angle quality inspection. It can be considered that the quality of grid meets the design requirements well. After grid independence test, the number of grid is 3,200 thousand, the minimum grid distance near the wall surface is $0.2 \mathrm{~mm}$, and the hexahedral mesh diagram is shown in Fig. 11.

Fig. 12 shows contours for Mach number 2.5 for isometric and front view. Angle of attack is zero.

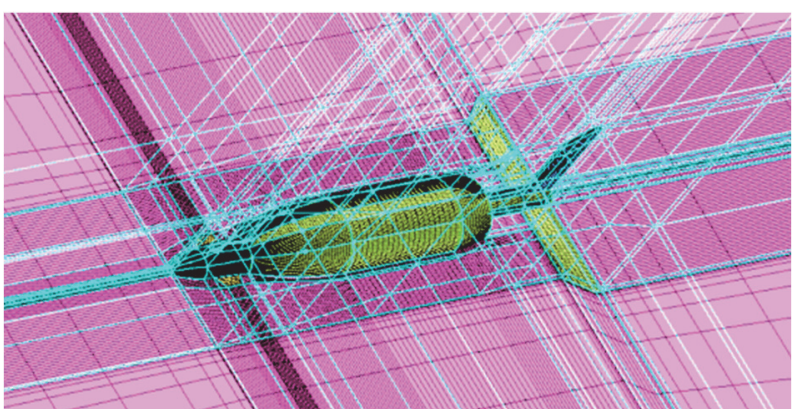

(a)

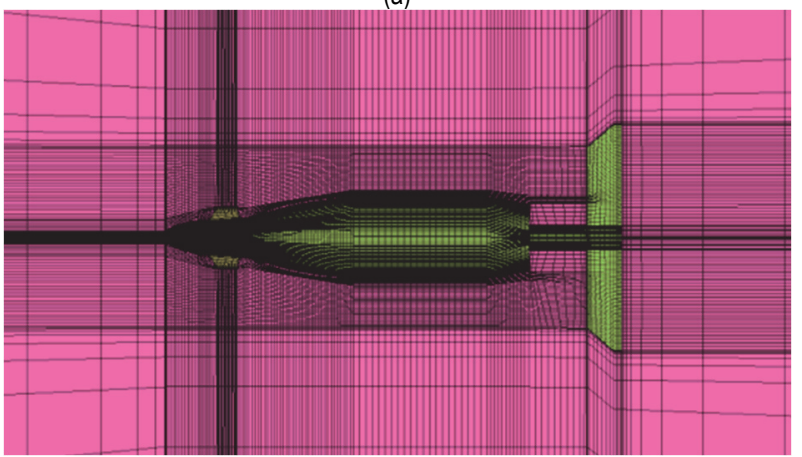

(b)

Figure 11 The sketch of a hexahedral mesh outflow field: (a) isometric and (b) front view

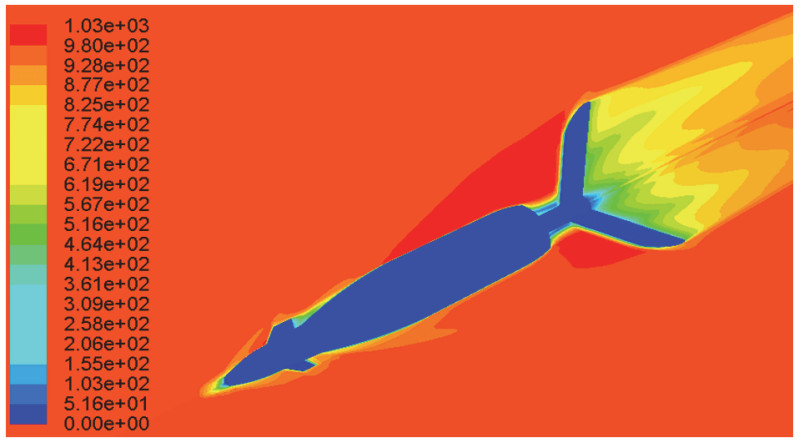

(a)

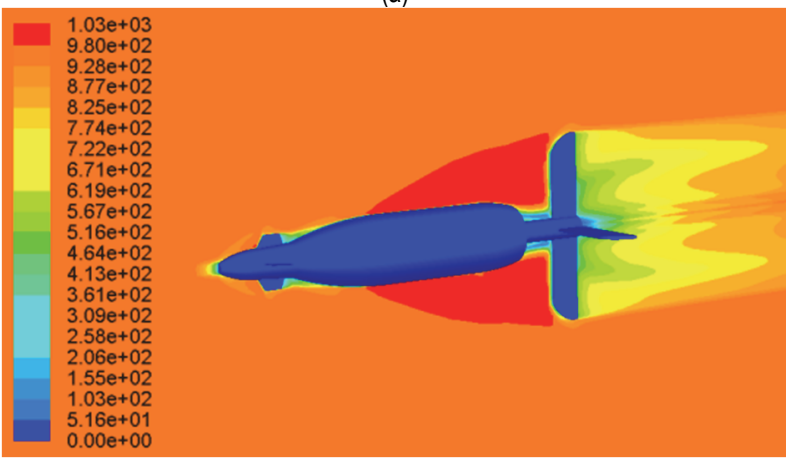

(b)

Figure 12 contours around projectile, Mach = 2.5: (a) isometric and (b) front view

\section{CONCLUSION}

This paper established a parametric modeling system of the trajectory correction ammunition. The problems and solutions in the parameterized simulation modeling are analyzed. The script design connects the model with the parameterization of the grid, and forms a whole set of intelligent ammunition design methods. The development cycle of the structure and the external ballistic control algorithm of this kind of ammunition has been speeded up, by adopting the parameterization design method on the 
modified ammunition. It is proved that parameterized modeling is not only academic research, but also has important practical significance in engineering application.

\section{REFERENCES}

[1] Xie, B.-Z., Jiang, Q.-M., \& Xu, W. (2016). Parametric CFD mesh generation of exhaust pipe based on ICEM.Computer Applications, 35(11), 37-39.

[2] Hao, Y., Guo, Y., \& Zhang, J. (2013).The study of aerodynamic layout for two-dimensional trajectory correction projectile canard rudder device.Journal of Projectiles, Rockets, Missiles and Guidance, 33(6), 121-124.

[3] Cheng, J., Yu, J.-Y., \& Wang, X.-M. (2016). Engineering modeling and identification of aerodynamics of trajectory correction projectile with decoupled canards. Acta Armamentarii, 37(10), 1812-1819.

[4] Yilmaz, E. \& Aliabadi, S. (2013). Application of surface conformed linear mesh and data subdivision technique to a spinning projectile.Computers \& Fluids, 88, 773-781. https://doi.org/10.1016/j.compfluid.2013.06.006

[5] Xie, K.-F., Zhang, H., \& Tang, Y.-F. (2015). Two-dimension correction strategy and capability for retractable canard. Journal of Chinese Inertial Technology, 23(4), 477-482.

[6] Haselbacher, A. \& Blazek, J. (2000). Accurate and Efficient Discretization of Navier-Stokes Equations on Mixed Grids. AIAA Journal, 38(11), 2094-2012. https://doi.org/10.2514/2.871

[7] Mifsud, M. J., Shaw, S. T., \& MacManus, D. G. (2010). A high-fidelity low-cost aerodynamic model using proper orthogonal decomposition. International Journal for Numerical Methods in Fluids, 63, 468-494. https://doi.org/10.1002/fld.2085

[8] Thomas, P. D. \& Lombard, C. K. (1979). Geometric Conservation Law and Its Application to Flow Computations on Moving Grids. AIAA Journal, 17(10) 1030-1037. https://doi.org/10.2514/3.61273

[9] Liu, W., Wie, Z., \& Wang, Z. (2018). The Design of Parametric Modeling of Rotary Locking Mechanism Based on the Secondary Development of UG. Ordnance Industry Automation, 37(04), 42-46. https://doi.org/10.1016/j.automatica.2018.09.021

\section{Contact information:}

\section{Wei ZHOU}

School of Equipment Engineering,

Shenyang Ligong University,

Shenyang 110159, Liaoning, China

College of Energy and Water Resources,

Shenyang Institute of Technology 113122, Liaoning, China

\section{Jian ZHANG}

(Corresponding author)

School of Equipment Engineering,

Shenyang Ligong University,

Shenyang 110159, Liaoning, China

E-mail:wilchou724@126.com

\section{Qingjie NI}

Liaoshen Industries Group Co., Ltd.,

Shenyang 110045, Liaoning, China

\section{Yufeng $\mathrm{HAO}$}

Liaoshen Industries Group Co., Ltd.,

Shenyang 110045 , Liaoning, China

\section{Yu LIANG}

Liaoshen Industries Group Co., Ltd.,

Shenyang 110045, Liaoning, China 